\title{
SIMULATION-BASED PLANNING OF MAINTENANCE ACTIVITIES IN THE AUTOMOTIVE INDUSTRY
}

\author{
Maheshwaran Gopalakrishnan \\ Anders Skoogh \\ Dept. of Product and Production Development \\ Chalmers University of Technology \\ SE 41296 Göteborg, SWEDEN
}

\author{
Christoph Laroque \\ Business Computing, esp. CIM \\ University of Paderborn \\ Paderborn, 33102, GERMANY
}

\begin{abstract}
Factories world-wide do not utilize their existing capacity to a satisfactory level. Several studies indicate an average Overall Equipment Efficiency (OEE) of around 55\% in manufacturing industry. One major reason is machine downtime leading to substantial system losses culminating in production plans with unsatisfactory robustness. This paper discusses an approach to integrate maintenance strategies into a production planning approach using discrete event simulation. The aim is to investigate how and where in the planning process maintenance strategies can be integrated and how different maintenance strategies influence production performance and the overall robustness of production plans. The approach is exemplified in an automotive case study, integrating strategies for reactive maintenance in a simulation model to support decision making on how repair orders should be prioritized to increase production performance. The results show that introducing priority-based planning of maintenance activities has a potential to increase productivity by approximately $5 \%$.
\end{abstract}

\section{INTRODUCTION}

Capacity issues and utilization of existing production resources are naturally central items at the development agenda in manufacturing companies. High utilization should not be seen as a value itself but it is an important aspect in avoiding unnecessary investments and, thus, staying economically sustainable. However, there is also an ecological point in being resource-efficient since studies have shown that around $30 \%$ of the energy consumption in manufacturing industry is wasted on system losses (i.e. machines are waiting for each other in stand-by mode) (Skoogh, Johansson, and Hansson 2011). Therefore, it is problematic that the current utilization of production resources is only around 55\% (Ingemansson 2004; GoodSolutions AB 2012).

The authors' experiences from numerous studies of flow-oriented industrial manufacturing systems are that $10-20 \%$ of the losses are due to direct downtime (failures) in the individual machines and the remaining part mostly because of system losses (blocked and idle states). One reason to these figures is the increased use of flow-oriented layouts in industry. Flow-oriented layouts are selected because of lower lead-times, reduced material handling, easier planning, and higher amounts of value-added time compared to for example process-oriented layouts in which equipment utilization rate is low, in-process inventories are high and more attention needed for each product. However, flow-orientation leads to tighter connection between production resources and, thus, more sensitive systems.

To deal with the challenges of flow-oriented production systems, approaches like the Theory Of Constraints (TOC) have become important in production planning and development (Goldratt 1990). The cornerstones in TOC are: rigid bottleneck analysis and an iterative approach (see the list below) to managing the system and its development: 


\section{Gopalakrishnan, Skoogh, and Laroque}

1. Identify the system's constraint(s)

2. Decide how to exploit the system's constraint(s)

3. Subordinate other resources to the constraint(s)

4. Elevate the system's constraint(s)

5. If in any of the previous steps a constraint is broken, go back to step 1 .

From a maintenance perspective, TOC clearly stresses the importance of constantly tracking the system bottleneck and making sure that this resource has as little downtime as possible. In other words, the bottleneck should always be prioritized in favor of other resources. For example, if an operator is repairing a non-bottleneck machine and the bottleneck goes down, the operator should immediately stop his or her current activity and start repairing the bottleneck.

Is this how it works in industry today? Unfortunately, the answer is no according to the authors' observations. The planning and execution of maintenance activities is often based on a first-come-firstserved basis. Another company also (ironically) stated the term "acoustic planning," i.e. the one that screams the loudest gets help first. However, in some companies, the bottleneck is actually prioritized but the priority is often set already at the design stage of the production system and does not consider the dynamic behavior of system constraints. Thus, the fact that bottlenecks constantly move due to system changes, production planning, natural variations in downtime, etcetera is disregarded.

The bottom line is that there is a need for holistic views and systems thinking in the planning of service and maintenance activities, both preventive and reactive. There are a few research initiatives in this direction. A good example is ( $\mathrm{Ni}$ and Jin 2012) presenting five important aspects for efficient maintenance operations: data-driven bottleneck identification, identification of maintenance windows, prioritization of maintenance tasks, joint production and maintenance scheduling and maintenance staff management. However, more efforts are desired to support the development in this direction and to quantify the benefits of being more holistic and flow-oriented the planning of service and maintenance activities.

The aim of this paper is to quantify the effects of applying a flow-based planning of reactive maintenance activities compared to a first-come-first-served approach. The main idea is to evaluate the impact of always prioritizing the repair orders for system constraints and also let the operators interrupt activities in non-bottlenecks in order to immediately serve the system constraint. As additional experiments, the paper also evaluates possible benefits of changing operator responsibilities to level the workload in the production flow; see the experimental planning in section 3.2.

This introduction is followed by a review of previous work on simulation studies in maintenance operations and various bottleneck detection methods. Thereafter, there is an outline of the research methodology used, including description of the automotive case study and the improvement scenarios evaluated in the simulation model. The results of model building and the simulation-based evaluation of improvement scenarios are presented before a concluding discussion about future work about integrating the gained knowledge in an simulation-based optimization approach for robust production planning.

\section{FRAME OF REFERENCES}

\subsection{Maintenance and Repair in Production Systems}

Maintenance and repair are integral and unavoidable occurrences in a production system. Many decision support systems for both reactive and preventive maintenance have been researched. The model-based maintenance decision support systems are needed to achieve high productivity and cost effectiveness (Ni and Jin 2012). Prioritization of the maintenance and repair provides improvement in the overall throughput of the system. Maintenance task prioritization by Value Based Maintenance Order (VMO) (Altuger and Chassapis 2009) and System Value Based (SVB) (Ni and Jin 2012) method use values from the machines and finds an optimized maintenance priority. With respect to repairs it is very important to understand about bottlenecks and detect where they are in the system. 


\section{Gopalakrishnan, Skoogh, and Laroque}

\subsection{Bottleneck Detection}

To improve the throughput of the system, the throughput of the bottlenecks has to be improved (Goldratt 1990). There are different traditional bottleneck detection techniques like the Machine Utilization method, waiting time method (Roser, Nakano, and Tanaka 2003) and theoretical bottleneck detection. New and more effective bottleneck detection methods are developed for short-term and long-term bottleneck detection using data-driven bottleneck detection ( $\mathrm{Li}$, Chang, and $\mathrm{Li}$ 2009), a shifting bottleneck detection method (Roser, Nakano, and Tanaka 2003) which shows there are multiple bottlenecks in a production system at a given point of time, and a method using inter-departure time and failure cycle data which detects and ranks the bottleneck (Sengupta, Das, and VanTil 2008).

\subsection{Simulation in Service Maintenance}

Discrete event simulation (DES) is applied to various fields and so to the area of maintenance, mainly due to its ability to model stochastic changes in flexible systems. Current production systems in industry are simulated using DES. The bottleneck detection methods and maintenance and repair strategies are tested upon these simulated models. There are several examples of how DES has been used for improving service and maintenance operations. (Ali et al. 2008) analyzed optimized maintenance design for manufacturing performance improvement using simulation. (Altuger and Chassapis 2009) used Arena-based simulation modeling for Multi Criteria Preventive Maintenance scheduling. A Multi-Stage DES approach for Scheduling of Maintenance activities in a Semi-Conductor manufacturing line was carried out by (Scholl et al. 2012).

\section{METHODOLOGY}

In this paper, the evaluation of maintenance strategies and their impact on system losses and performance is based on one single case study in automotive industry. A DES approach is applied in order to compare

different planning strategies for maintenance activities to each other and to the current state model of the investigated system. The simulation study followed the traditional steps of a simulation project, e.g. described in (Banks 1996) and later also updated with a more parallel work procedure in (Rabe, Spieckermann, and Wenzel 2008).

\subsection{Use-Case Description}

The case study company produces engine components to cars and the sub-system under investigation is delimited to one specific manufacturing line. There are 11 serially connected operations including 15 machines (Figure 1). The machines mainly execute machining operations such as multi-operational rough machining, milling, drilling, and grinding. Unpacking of raw material, washing, quality control and visual inspection are example of other steps in the production system. Some of the operations are connected with conveyor belts acting as buffers, nine in total. Production planning follows a push principle and the overall goal is to produce as many products as possible per time unit.

Four operators work at the production line and they are responsible for separate areas of the line. Operator $1(\mathrm{O} 1)$ is responsible for machines M1 and M2(1) to M2(2), O2 handles machines M2(3) to M2(5), O3 handles machines M3 through M6 and finally O4 handles machines M7 to M11. The operator responsibilities include set-ups between product variants, quality control, and most importantly for this case study, the repair of common failures. Repair activities are currently executed on a first-come-first-served basis. The operator maintenance takes care of most problems in the machines and external assistance from the maintenance department is rare for reactive maintenance. 


\section{Gopalakrishnan, Skoogh, and Laroque}

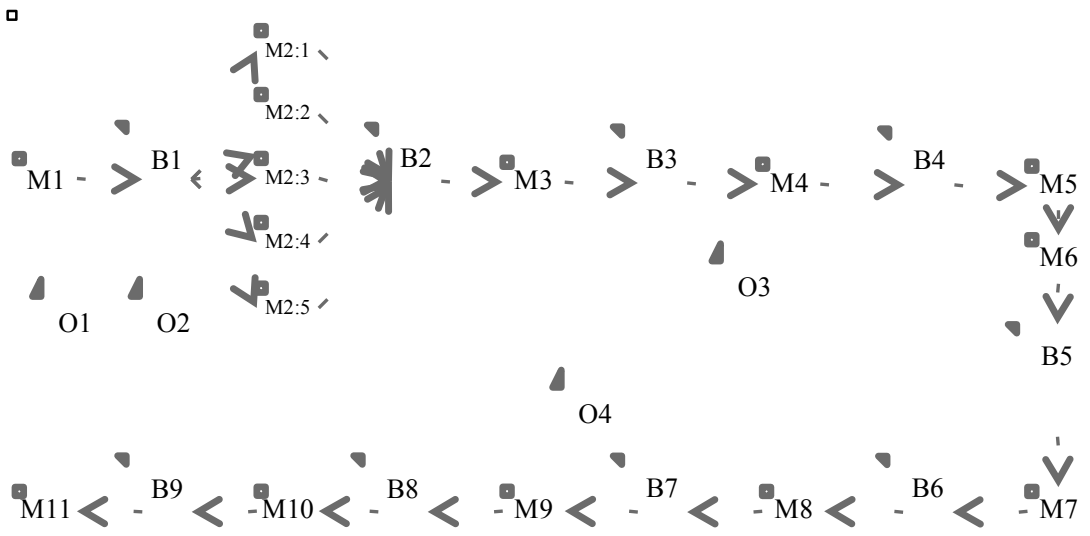

Figure 1: Factory Layout ( $\mathrm{M}=$ Machine, $\mathrm{B}=\mathrm{Buffer}, \mathrm{O}=$ Operator $)$

A conceptual model (Van der Zee and Van der Vorst 2007), describing system behavior, was created using observations and interviews with operators and process experts. Quantitative data, e.g. cycle times and down times, were collected mainly from computerized data sources at the case study company. Cycle times are created from spreadsheets by the production engineers and downtimes (start times and durations for failures) are populated online from a database with failure reports from the operators. All data were cross-checked with qualitative methods such as interviews and work-shops with process experts. After data processing and distribution fitting, the description of a system entity could look like the following example (Figure 2):

The first operation in the line is called M1 (Machine 1). This machine simply unpacks raw material from pallets and put them onto the $\mathrm{MH}$ equipment for further transportation throughout the line.

Cycle time $\mathrm{M} 1=$ constant $7 \mathrm{sec}$

MTTF M1 = exponential with mean $150 \mathrm{~min}$

MTTR M1 = Erlang distributed with mean 18 min and shape $=2$.

Maintenance and set-ups require operator $1(\mathrm{O} 1)$

Figure 2: Example Operation

\subsection{Experimental Plan}

As mentioned in the introduction to this paper, different maintenance planning strategies were evaluated by comparing a set of scenarios. Each scenario was implemented as a separate simulation model.

Base Model: The current state model with planning on a first-come-first-served basis (reference model). Scenario 1: Operators prioritize repair orders based on the machine's importance for the production flow. Repair work at one machine is interrupted, if a machine with higher priority breaks down. A priority list is established based on bottleneck analysis of the current state model.

Scenario 2: The operator work load is leveled by changing responsibility areas for the operators.

Scenario 3: An "optimal" situation where the operators act as a team and they are all trained for repairing all machines.

The work procedure started with modeling the current state and proceeded with a thorough bottleneck analysis using the same model. Thereafter, each scenario was implemented, simulated, and compared to the reference model. All simulations used a warm-up time of 8 hours, a run length of 1 working week (168 hours), and were run using 10 replicates in order to collect the necessary results for analysis. 


\section{SIMULATION MODEL}

\subsection{Model Building}

The production system described in section 3.1 was modeled using Automod®. All machines and operators were modeled as individual model entities and coded in separate procedures. The breakdown behavior of production resources were also coded separately in the source file to reach full flexibility when experimenting with the various maintenance strategies. A graphical representation of the model is shown in Figure 3. Not much time and efforts have been spent on 3D-graphics because impressive visualization does not add substantial value to the aim and questions of this study. Animation has been used mostly for verification purposes.

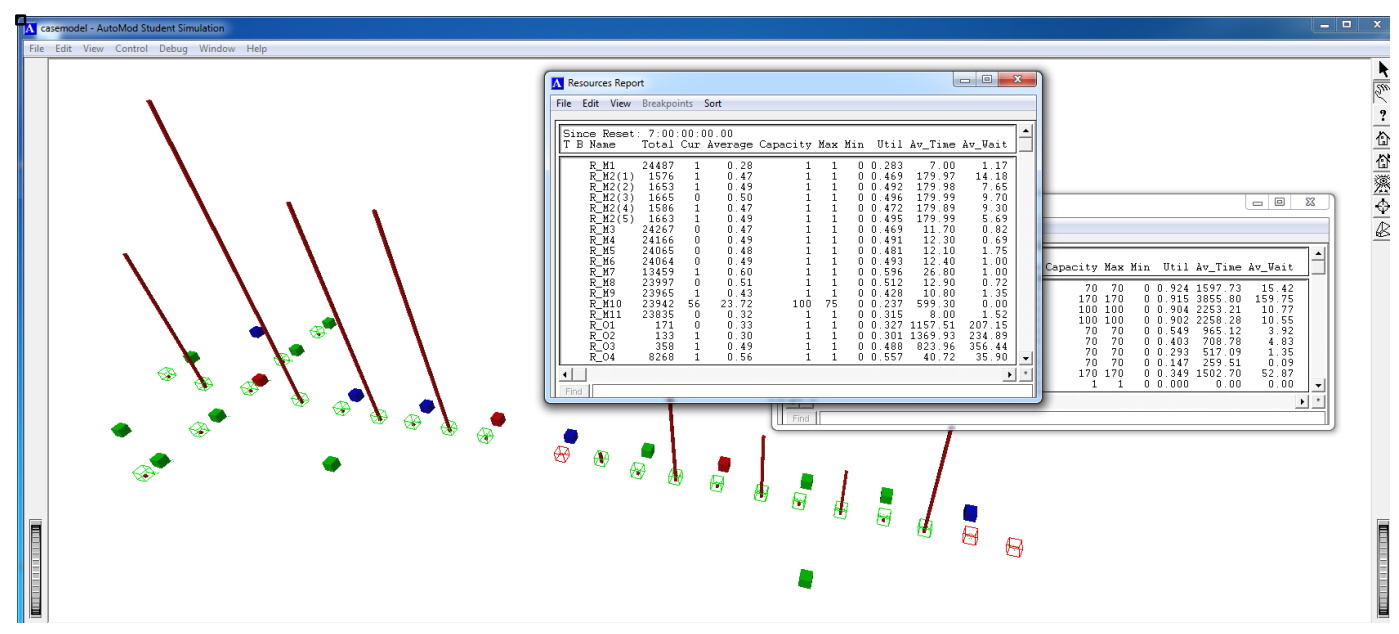

Figure 3: Graphical Representation of the Model

\subsection{Abstraction Level}

The importance of selecting a feasible level of detail for the simulation model is often stressed in literature (Vasudevan and Devikar 2011). This model is designed to evaluate scenarios regarding reactive maintenance and the impact of operator priorities in such a context. Therefore, the major input data to the model entities are the machine cycle times, their Mean Time To Failure (MTTF), and Mean Time To Repair (MTTR). Data and logics describing scrap rates, quality control on a machine level, set-up times, etcetera are excluded. The main assumptions made in this model are:

- The work of the operators is limited to reactive maintenance efforts.

- The model is delimited to operator maintenance and excludes failures whose repair requires external competence.

- Only the major product variant is included in the model.

- Suppliers are excluded from the model and raw materials are considered always available.

- Customer orders are unlimited and all produced parts can be sold.

- The material handling equipment (i.e. the conveyors between machines) is considered a nonbottleneck and, thus, the conveyor belts are replaced with time-delayed buffers.

- The plant is operating on 5 shifts (168 hours/week) and operator breaks are not modeled.

- No transfer times between operators for repair orders

\subsection{Verification \& Validation}

The verification of the base model was done by using the debugging function and animation environment provided in Automod ${ }^{\circledR}$. The code and simulation results were also cross-checked by two model builders from the research team. Furthermore, the changes from the base model to the different experimental sce- 


\section{Gopalakrishnan, Skoogh, and Laroque}

narios were verified by comparing relevant statistics from the model entities, e.g. making sure that resource utilizations corresponded or changed in expected patterns. Validation of the base model was performed by ensuring correspondence between the model results (mainly production output) and the realworld system. Since this was done by a process expert, the validation procedure can be categorized as face validation (Sargent 2005). The validation procedure revealed a previous problem in data collection. The downtimes, originally collected from the database, included waiting times for the operators to start repairing a machine, counted from the time that it actually broke down. These waiting times had to be subtracted from the MTTR data because the operator's behavior for maintenance-related activities (including waiting times) are handled by the model logics.

\section{RESULTS AND SCENARIO ANALYSIS}

\subsection{Base Model Results}

In order to have a reference model for comparing the impact of the maintenance strategies described in section 3.2 as scenarios, the first step is to collect results from the base model. The base model is the current state of the investigated production system described in section 3.1. Table 1 shows working time, downtime, utilization, idle \& blocked (theoretical and actual) in percentage for the base model.

Table 1: Base Model's Utilization and Idle \& Blocked times

\begin{tabular}{|c|c|c|c|c|c|c|}
\hline Machine & Working \% & Down Time \% & Utilization \% & Idle \& Blocked \% & $\begin{array}{c}\text { Theoretical } \\
\text { Utilization \% }\end{array}$ & $\begin{array}{c}\text { Theoretical Idle \& } \\
\text { Waiting \% }\end{array}$ \\
\hline M1 & 28.54 & 13.2 & 41.74 & 58.26 & 30.25 & 69.75 \\
\hline M2(1) & 48.58 & 14.2 & 62.78 & 37.22 & 51.88 & 48.12 \\
\hline M2(2) & 48.77 & 14.5 & 63.27 & 36.73 & 52.09 & 47.91 \\
\hline M2(3) & 48.88 & 13.9 & 62.78 & 37.22 & 52.19 & 47.81 \\
\hline M2(4) & 49.04 & 13.8 & 62.84 & 37.16 & 52.40 & 47.59 \\
\hline M2(5) & 49.46 & 13.1 & 62.56 & 37.44 & 52.81 & 47.19 \\
\hline M3 & 47.67 & 17 & 64.67 & 35.33 & 49.80 & 50.20 \\
\hline M4 & 50.08 & 14.9 & 64.98 & 35.02 & 52.34 & 47.66 \\
\hline M5 & 49.29 & 21.1 & 70.39 & 29.61 & 55.23 & 44.77 \\
\hline M6 & 50.51 & 19.3 & 69.81 & 30.19 & 55.04 & 44.96 \\
\hline M7 & 60.54 & 12.2 & 72.74 & 27.26 & 63.17 & 36.83 \\
\hline M8 & 52.57 & 19.5 & 72.07 & 27.93 & 57.25 & 42.75 \\
\hline M9 & 44.06 & 18 & 62.06 & 37.94 & 48.19 & 51.81 \\
\hline M10 & 24.48 & 3.3 & 27.78 & 72.22 & 24.52 & 75.48 \\
\hline M11 & 32.64 & 15.2 & 47.84 & 52.16 & 34.18 & 65.82 \\
\hline
\end{tabular}

Then in Table 2, buffers and their average waiting times and queue lengths are shown. After 25 successive replications of the base model the average throughput of the system is 24,681 loads per 168 hours, with a standard deviation of 1024 loads.

Table 2: Base Model's Queue Waiting Time and Queue Length

\begin{tabular}{|c|c|c|}
\hline Queues & Avg Waiting Time & Avg Queue Length \\
\hline B1 & 1569.72 & 64.037 \\
\hline B2 & 3759.13 & 153.85 \\
\hline B3 & 2250.71 & 91.866 \\
\hline B4 & 2250.71 & 88.896 \\
\hline B5 & 1011.07 & 41.247 \\
\hline B6 & 761.13 & 31.052 \\
\hline B7 & 501.77 & 20.489 \\
\hline B8 & 245.64 & 10.019 \\
\hline B9 & 1602.28 & 65.529 \\
\hline
\end{tabular}




\subsubsection{Bottleneck Detection in the Base Model}

From the base model results, the bottleneck of the production system is analyzed using following methods:

i) Utilization Method:

The utilization of a machine is the time during which the machine is active, comprising working, in repair, changing tools or servicing (Roser, Nakano, and Tanaka 2003). Therefore the machine utilization here is the sum of the working and downtimes. Figure 4 shows that machine M7 has the highest active time, which suggests that it has high probability of being the bottleneck of the overall system. Furthermore, machines M8, M5, and M6 are also interesting in given order.

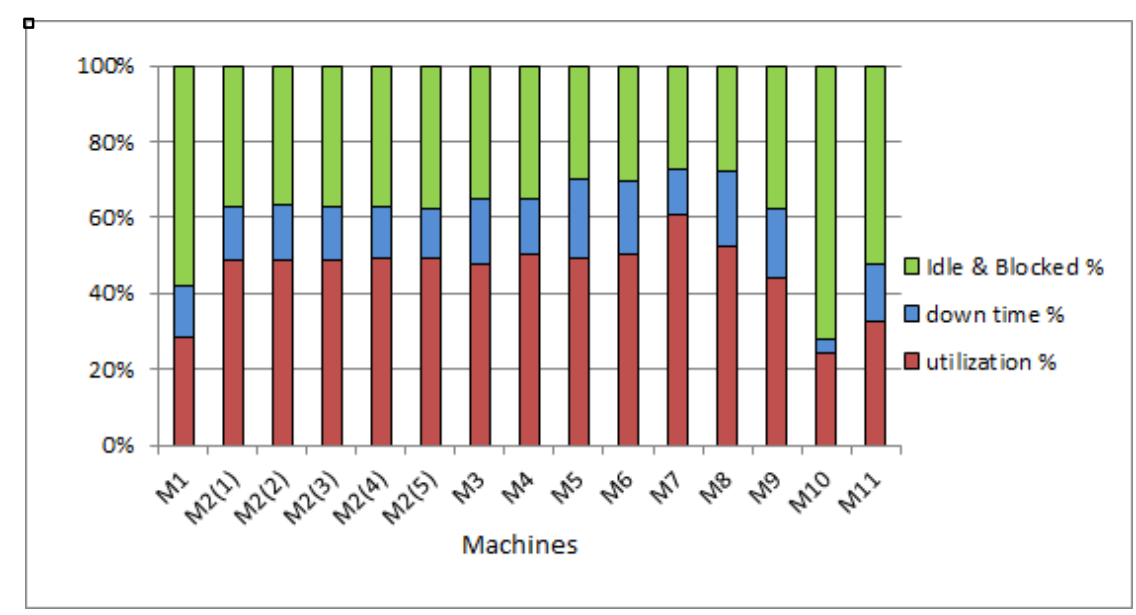

Figure 4: Utilization of resources in the base model

ii) Waiting Time Method:

From Figure 5 and Figure 6 it can be observed, that there is a sharp decrease in queue length and queue waiting time from B4 to B5, which suggests that the machine after buffer B5(M7) is the bottleneck of the overall system. Similarly note, because of decrease in the queue length B2 to B3 the machine after B2 (M3) is a probable bottleneck of the overall system.

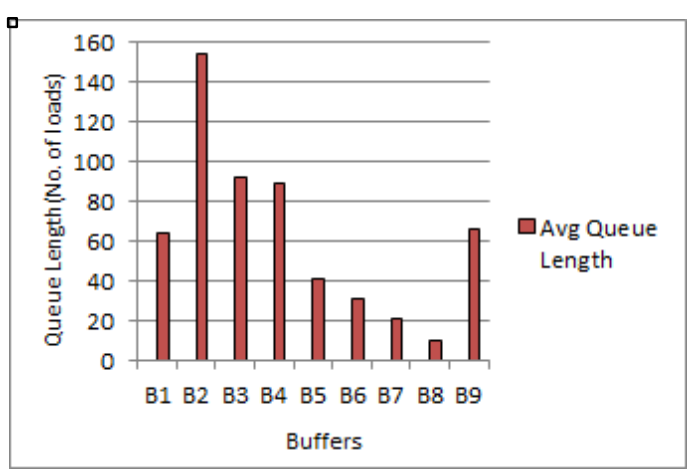

Figure 5: Queue Length

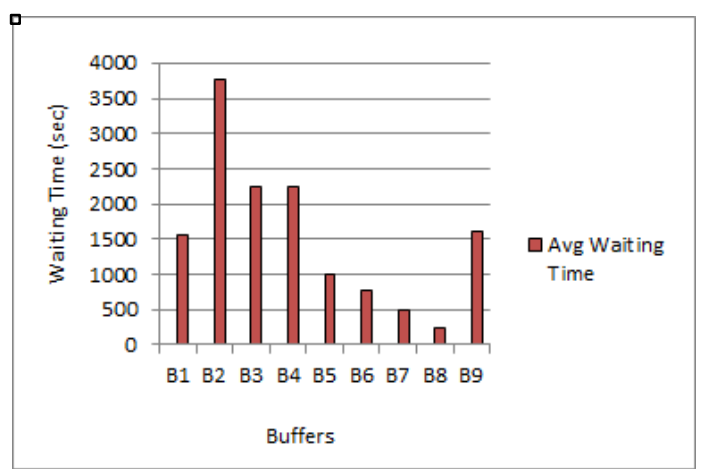

Figure 6: Waiting Time

iii) Theoretical Utilization Method:

The theoretical utilization method suggests the bottleneck machine similarly to the utilization method, but machine utilization is theoretically calculated from the input data to the base model. The percentage Utilization and Idle \&Wait time is shown in Table 1. The graphs looks very simi- 
lar to Figure 4, therefore by theoretical method as well, machine M7 is the bottleneck of the overall system.

Currently these are the most common bottleneck detection methods employed in the industries. Each method has its own advantages and disadvantages, e.g. the problem with different queue capacities described in (Roser, Nakano, and Tanaka 2003). As a result of this bottleneck analysis a Priority List is created (see Table 3 ). This priority suggests criticality of the machines with respect to bottlenecks. Machine M1 and M2(1) to M2(5) is excluded, as M1 is the starting machine and all M2 are identical machines.

Table 3: Priority List

\begin{tabular}{|c|c|c|c|c|c|c|c|c|c|}
\hline Priority & 1 & 2 & 3 & 4 & 5 & 6 & 7 & 8 & 9 \\
\hline Machine & M7 & M8 & M5 & M6 & M4 & M3 & M9 & M11 & M10 \\
\hline
\end{tabular}

This paper suggests 3 different scenarios for the production system to improve its throughput by strategically working on the reactive maintenance of the production system.

\subsection{Scenario 1: Prioritize Repair Orders}

In this scenario the machines are given priority for repair activities according to the bottleneck machines found using the detection methods applied to the base model. The machines are prioritized to individual operators rather than the entire production system. For this scenario the operators' responsibilities are the same as the base model. Operators O1 and O2 work on M1, M2(1) through M2(5). From the bottleneck analysis, both these machines are non-bottlenecks. Therefore, prioritization of these machines is excluded.

For operator 4, M7 is the direct bottleneck from all the detection methods, therefore it gets priority 1 and the other machines get priorities with respect to the utilization percentages of individual machines. In case of operator O3, M5 gets priority 1 as it is a critical machine and it does not follow up with a buffer in between with its next machine M6. On that note M6 gets priority 2. But according to the queue length method M3 seems to be a probable bottleneck machine, therefore 2 trials are made with M3 being priority 3 and the other with M4 being priority 3. Table 4 shows the two different trials employed.

Table 4: Trial $1 \& 2$

\begin{tabular}{|c|c|c|c|}
\hline Operator & Priority & Trial 1 & Trial 2 \\
\hline \multirow{3}{*}{ O3 } & 1 & M5 & M5 \\
\cline { 2 - 4 } & 2 & M6 & M6 \\
\cline { 2 - 4 } & 3 & M3 & M4 \\
\cline { 2 - 4 } & 4 & M4 & M3 \\
\hline \multirow{3}{*}{ O4 } & 1 & M7 & M7 \\
\cline { 2 - 4 } & 2 & M8 & M8 \\
\cline { 2 - 4 } & 3 & M9 & M9 \\
\cline { 2 - 4 } & 4 & M11 & M11 \\
\cline { 2 - 4 } & 5 & M10 & M10 \\
\hline
\end{tabular}

From the throughput results (further detailed in section 5.5), Trial 1 gives an average improvement of 4.6\% on the overall throughput of the system when compared to the base model. The case described in Trial 2 gives an average improvement of $\mathbf{5 . 1 \%}$ on the overall throughput of the system when compared to the base model.

\subsection{Scenario 2: Changing Workload of the Operators}

In this scenario, the paper suggests rearranging the responsibilities of the operators. Different alternatives of changed workload for the operators were tried and the one which gave the highest throughput is sug- 


\section{Gopalakrishnan, Skoogh, and Laroque}

gested in Table 5, with a comparison to the base model's operator workload distribution. From the throughput results (see section 5.5 for details), it is observed that the change in responsibility of the operators gives an average improvement of $\mathbf{4 . 9 \%}$ on the overall throughput of the system when compared to the base model.

Table 5: Base Model's and Scenario 2's Operator Workload Distribution

\begin{tabular}{|c|c|c|}
\hline Operator & Base Model & Scenario 2 \\
\hline $\mathrm{O} 1$ & $\mathrm{M} 1, \mathrm{M} 2(1)$ to $\mathrm{M} 2(2)$ & $\mathrm{M} 1, \mathrm{M} 2(1$ to 5$)$ \\
\hline $\mathrm{O} 2$ & $\mathrm{M} 2(3)$ to $\mathrm{M} 2(5)$ & $\mathrm{M} 3, \mathrm{M} 4$ \\
\hline $\mathrm{O} 3$ & $\mathrm{M} 3, \mathrm{M} 4, \mathrm{M} 5, \mathrm{M} 6$ & $\mathrm{M} 5, \mathrm{M} 6, \mathrm{M} 7, \mathrm{M} 8$ \\
\hline $\mathrm{O} 4$ & $\mathrm{M} 7, \mathrm{M} 8, \mathrm{M} 9, \mathrm{M} 10, \mathrm{M} 11$ & $\mathrm{M} 9, \mathrm{M} 10, \mathrm{M} 11$ \\
\hline
\end{tabular}

\subsection{Scenario 3: Work Team Solution}

This scenario tries to give an even better solution, where all the operators work together as a centralized team and anyone can repair any machine. Hence it is a prerequisite for the operators to have knowledge of all the machines. The machine which breaks down will be repaired by any operator who is free. From the throughput results (see section 5.5 below), it is observed that there is an average improvement of $\mathbf{1 1 . 2 \%}$ on the overall throughput of the system when compared to the base model.

\subsection{Throughput Results and Comparison}

As mentioned in experimental plan (section 3.2), the base model as well as the simulations of the three improvement scenarios are run for 25 replications. The mean throughputs along with standard deviation for all scenarios are tabulated in Table 6.

Table 6: Throughput Results

\begin{tabular}{|c|c|c|}
\cline { 2 - 3 } \multicolumn{1}{c|}{} & Mean Throughput & Standard Deviation \\
\hline Base Model & 24681 & 1165.9 \\
\hline Scenario 1 (Trial 1) & 25806 & 760.1 \\
\hline Scenario 1 (Trial 2) & 25940 & 1169.2 \\
\hline Scenario 2 & 25893 & 881.31 \\
\hline Scenario 3 & 27447 & 1084.4 \\
\hline
\end{tabular}

Interval estimations for all scenarios were also calculated based on mean and standard deviations from the 25 replications. From Figure 7 it is evident that the throughput levels of the scenarios do not overlap with the base model. The improvements obtained from the scenarios are therefore realistic and significant. Intervals are calculated at $95 \%$ confidence level. 
口

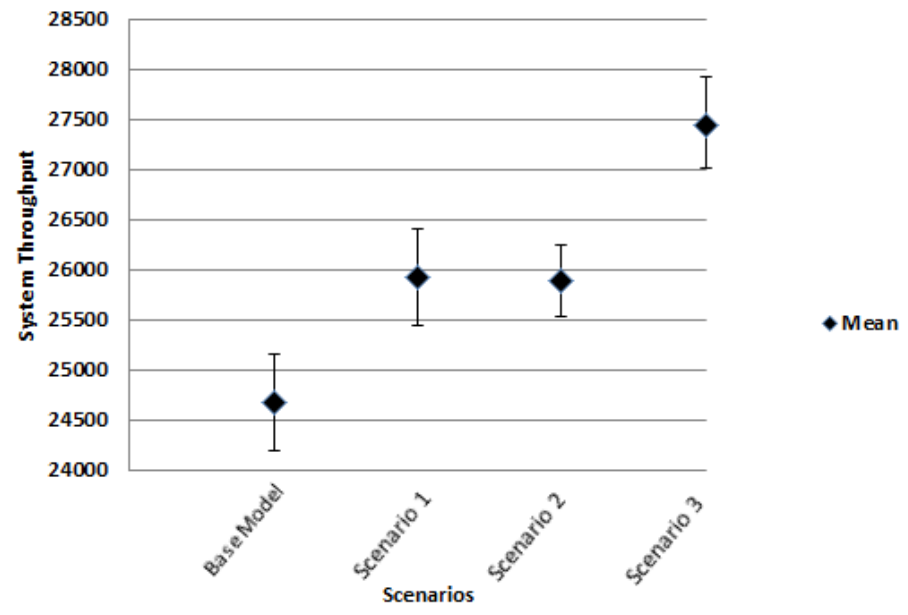

Figure 7: Throughput Comparison

\section{DISCUSSION OF FUTURE STEPS - INTEGRATION OF MAINTENANCE AND REPAIR IN ROBUST PRODUCTION PLANNING}

This study proved that a priority-based approach for the planning of reactive maintenance activities has substantial potential to increase productivity in manufacturing industry. For this specific case study in automotive industry, the productivity increased by around $5 \%$ only by applying a thorough bottleneck analysis and working smart from a production system viewpoint. In other words, no investments in personnel or equipment are required and the direct repair times are also the same as in the current state scenario (the base model). What might be necessary to put these results into practice is clever decision support tools for the operators to receive downtime alarms and assistance in the priorities among machines in real-time. It should also be mentioned that this study uses a long-term approach in the detection of bottlenecks and, thus, resulting in static priority rules. An interesting future step is to implement dynamic bottleneck detection methods (Roser, Nakano, and Tanaka 2003) and enable constant updates of the maintenance priorities based on real-time information and current status of the production system.

Another interesting step for future research is the integration of maintenance aspects in production planning using simulation-based optimization. In (Laroque 2011) and (Laroque 2012), the use of the DES-based material flow simulation is proposed in order to evaluate the robustness of a production plan, which was created and optimized with no respect to unforeseen derivations. Since the necessary probabilities for machine failures and similar operational events on the floor can easily be integrated in the simulation model, in order to analyze, how initial plan performs in these situations. The influence of unforeseen events in daily production cannot be modeled within mathematical optimization without consuming large amounts of computation time. A possible way to use simulation to evaluate and enhance a production plan is shown and illustrated by using a real-world use-case of medium complexity.

The mathematical optimization model is connected with a down-streamed material flow simulation for this purpose. While always optimal conditions are assumed within the mathematical optimization model, the uncertainties of the production system are integrated by the simulation process. This allows an analysis whether a production plan is able to perform well creating an acceptable monetary solution under these changed conditions or not. It is easily possible to develop a more robust production plan with these tools. Simulations usually are used to verify the solutions of an optimization problem. However, the aim of this research is to replace parts of the planning process with simulation methods to receive solutions with an acceptable quality on a timely matter.

Maintenance and repair aspects have not yet been taken into consideration in these existing approaches and are to be integrated in the next steps. An open research question beside the optimal selection of a maintenance strategy during planning, is where to integrate the consideration of the maintenance issues technically: whether in the mathematical optimization in order to allow a more robust production plan al- 


\section{Gopalakrishnan, Skoogh, and Laroque}

ready integrating all substantial maintenance issues or to integrate this aspect within the simulation model during the evaluation phase. This further integration can answer the question, by which repair strategy the optimal production plan can stay robust for a longer time period and at which benefits and throughput rates.

\section{CONCLUSION}

This paper presents an approach for the improvement of productivity in manufacturing industries by selecting the best maintenance strategy for flow-shop factories. It is validated by an automotive case study, that a priority-based planning of maintenance activities improved productivity by approximately $5 \%$ for a specific manufacturing line, which is without additional efforts. Additional simulation analyses also showed the possibility to increase productivity even more up to $11 \%$ by changing operator responsibilities or all operators work as a team. However, such changes are considered more challenging to implement compared to the main, priority-based, scenario. Based on these results, further work is discussed in brief, that will include the underlying assumptions in an simulation-based approach for robust production planning.

\section{ACKNOWLEDGMENTS}

Parts of this work has been performed within the Sustainable Production Initiative and the Production Area of Advance at Chalmers. The support is gratefully acknowledged.

\section{REFERENCES}

Ali, A., Chen, X., Yang, Z., Lee. J, Ni, J. 2008. "Optimized Maintenance Design for Manufacturing Performance Improvement using Simulation". In: Proceedings of the 2008 Winter Simulation Conference, edited by S.J. Mason, R.R. Hill, L. Mönch, O. Rose, T. Jefferson, J.W. Fowler, 1181-1819.

Altuger, G., C. Chassapis. 2009. "Multi criteria preventive maintenance through Arena based simulation modeling". In: Proceedings of the 2009 Winter Simulation Conference, edited by M.D. Rossetti, R.R. Hill, B.Johansson, A.Dunkin and R.G. Ingalls, 2123-2134.

Banks, J., J.S. Carson, and B.L. Nelson. 1996. Discrete-Event System Simulation (2nd ed.), Prentice-Hall, Upper Saddle River, New Jersey.

Goldratt, E.M. 1990. What is This Thing Called Theory of Constraints and How Should it be Implemented? New York: North River Press.

GoodSolutions AB. 2012. Good Solutions customer survey. Presented at the FFI production research conference (In Swedish): Mötesplats för framtidens framgångsrika verkstäder, Katrineholm, Sweden, 2224 May 2012.

Ingemansson, A. 2004. On Reduction of Production Disturbances in Manufacturing Systems Based on Discrete-Event Simulation. PhD Thesis, Department of Mechanical Engineering, Lund University, Lund, Sweden.

Laroque, C., R. Delius, J.H. Fischer, D. Horstkaemper. 2012. "Increase Of Robustness In Production Plans Using A Hybrid Optimization And Simulation Approach", In: International Journal on Advances in Systems and Measurements, IARIA Xpert Publishing.

Laroque, C., R. Delius, J.H. Fischer, D. Horstkaemper. 2011. "Increase Of Robustness On Pre-Optimized Production Plans Through Simulation-Based Analysis And Evaluation". In: Proceedings of The Third International Conference on Advances in System Simulation. IARIA, Xpert Publishing Services

Li, L., Q. Chang, J. Ni. 2009. "Data Driven Bottleneck Detection of Manufacturing Systems". In: International Journal of Production Research. 47(18), 5019-5036.

$\mathrm{Ni}$, J., and X. Jin. 2012. "Decision support systems for effective maintenance operations". In: CIRP Annals - Manufacturing Technology 61:411-414. 


\section{Gopalakrishnan, Skoogh, and Laroque}

Rabe, M., S. Spieckermann, S. Wenzel. 2008. "A New Procedure Model for Verification and Validation in Production and Logistics Simulation". In: Proceedings of the 2008 Winter Simulation Conference, edited by S.J. Mason, R.R. Hill, L. Mönch, O. Rose, T. Jefferson, J.W. Fowler, 1717-1726.

Roser, C., M. Nakano, M. Tanaka. 2003. "Comparison of Bottleneck Detection Methods for AGV Systems". In: Proceedings of the 2003 Winter Simulation conference, edited by S. Chick, P.J. Sanchez, D. Ferrin, and D.J. Morrice. 1192-1198

Sargent, R.G. 2005. "Verification and validation of simulation models". In: Proceedings of the 2005 Winter Simulation Conference, eds. M.E. Kuhl, N.M. Steiger, F.B. Armstrong, and J.A. Joines, 130-143.

Scholl, W., M. Mosinski, B.P: Gan, P. Lendermann. 2012. "A Multi-Stage Discrete Event Simulation Approach for Scheduling of Maintenance Activities in a Semiconductor Manufacturing Line". In: Proceedings of the 2012 Winter Simulation Conference, edited by C. Laroque, J. Himmelspach, R. Pasupathy, O. Rose, and A.M. Uhrmacher. 4673-4781

Sengupta,S., K. Das, R.R: VanTil. 2008. "A New Method for Bottleneck Detection". In: Proceedings of the 2008 Winter Simulation Conference, edited by S.J. Mason, R.R. Hill, L. Mönch, O. Rose, T. Jefferson and J.W. Fowler, 1741-1745

Skoogh, A., B. Johansson, and L. Hansson. 2011. "Data Requirements and Representation for Simulation of Energy Consumption in Production Systems". In: Proceedings of the 44th CIRP Conference on Manufacturing Systems 2011.

Van der Zee, D.-J. and J.G A.J. Van der Vorst. 2007. "Guiding principles for conceptual model creation in manufacturing simulation". In: Proceedings of the 2007 Winter Simulation Conference, edited by S.G. Henderson, B. Biller, M.-H. Hsieh, J. Shortle, J.D. Tew and R.R. Barton, 776-784.

Vasudevan, K., and A. Devikar. 2011. "Selecting Simulation Abstraction Levels in Simulation Models Of Complex Manufacturing Systems". In: Proceedings of the 2011 Winter Simulation Conference, edited by S. Jain, R.R. Creasey, J. Himmelspach, K.P. White, and M. Fu, 2273-2282.

\section{AUTHOR BIOGRAPHIES}

MAHESHWARAN GOPALAKRISHNAN is a PhD candidate working at the Department of Product and Production Development, Chalmers University of Technology, Sweden. He received his Masters in Production Engineering and Management from KTH, Royal Institute of Technology, Sweden in 2012. His main research area of focus is Decision Support for Service and Maintenance in Production Systems. His email address is mahgop@chalmers.se

ANDERS SKOOGH is an Assistant Professor in the area of Virtual Production at the Department of Product and Production Development, Chalmers University of Technology, Sweden. In 2011, he received his $\mathrm{PhD}$ in Production Systems from Chalmers. Before starting his research career, he accumulated industrial experience as a logistics developer at Volvo Car Corporation. His main research area is virtual tools for decision support in development of sustainable production systems. The current focus is on efficient management of production data, mainly for simulation and maintenance purposes. Anders is also the Director of Chalmers' Masters Programme in Production Engineering. His email address is anders.skoogh@chalmers.se.

CHRISTOPH LAROQUE studied business computing at the University of Paderborn, Germany. From 2003 to 2007 he has been a PhD student at the graduate school of dynamic intelligent systems and, in 2007, received his $\mathrm{PhD}$ for his work on multi-user simulation. He is team leader of the "simulation \& digital factory" at the chair of Business Computing, esp. CIM. He is mainly interested in the simulationbased decision support for operational production and logistic processes. His email address is laroque@upb.de. 